\title{
A FUNCTIONAL CALCULUS FOR SUBNORMAL OPERATORS
}

\author{
BY JOHN B. CONWAY ${ }^{1}$ AND ROBERT F. OLIN ${ }^{2}$ \\ Communicated October 24, 1975
}

\begin{abstract}
In this announcement some results concerning a functional calculus for subnormal operators, in which the functions involved are not necessarily continuous, are presented. This functional calculus can be used to study the structure of subnormal operators.
\end{abstract}

Throughout this paper $S$ is a subnormal operator on a separable Hilbert space $H, N$ is its minimal normal extension on $K$, and $\mu$ is a scalar spectral measure for $N$. If $T$ is any operator, $A(T)$ is the ultraweakly closed algebra generated by $T$ and $1, W(T)$ is the weak closure of $A(T)$, and $W^{*}(T)$ is the von Neumann algebra generated by $T$.

If $P^{\infty}(\mu)$ denotes the weak star closure of the polynomials in $L^{\infty}(\mu)$ then it is easy to show that $A(N)=W(N)=\left\{f(N): f \in P^{\infty}(\mu)\right\}$, and for every $f$ in $P^{\infty}(\mu), f(N)$ leaves $H$ invariant. Define $f(S)$ by the formula $f(S) x=f(N) x$ for each $x$ in $H$; equivalently, $f(S)=f(N) \mid H$.

THEOREM 1. $A(S)=\left\{f(S): f \in P^{\infty}(\mu)\right\}$.

In [1, p. 89] Bram showed that if $T \in W(S)$ then there is a unique $R$ in $W^{*}(N)$ such that $\|R\|=\|T\|, R H \subseteq H$, and $T=R \mid H$. He also asked if this could be strengthened to get that $W(S)=\{R \mid H: R \in W(N)\}$. Theorem 1 shows this to be possible if and only if $W(S)=A(S)$.

The next result has several applications in the study of subnormal operators.

THEOREM 2. If $(X, \Omega, \nu)$ is a finite measure space and $A$ is any weak star closed subalgebra of $L^{\infty}(\nu)$ that contains 1 , then there is a countable measurable partition $\left\{\Delta_{0}, \Delta_{1}, \ldots\right\}$ of $X$ with the following properties: (a) $\chi_{\Delta_{n}} \in A$ for all $n \geqslant 0$; (b) for $n \geqslant 1, \mathrm{~A}_{n} \equiv \mathrm{A} \chi_{\Delta_{n}}$ is antisymmetric (i.e., every hermitian element in $A_{n}$ is a multiple of $\chi_{\Delta_{n}}$ ); (c) $A_{0} \equiv A \chi_{\Delta_{0}}$ is pseudosymmetric (i.e., for every subset $\Delta$ of $\Delta_{0}$ with $\mu(\Delta)>0$ there is an element of $A_{0}$ that is real-valued on $\Delta$ but not constant there).

Using the methods of [2] (and, in particular, Theorem 1 of [2]), the following is obtained.

AMS (MOS) subject classifications (1970). Primary 47B20; Secondary 46J 10.

${ }^{1}$ During the preparation of this paper the first author was supported by National Science Foundation Grant No. 28601.

${ }^{2}$ Theorem 4 of this announcement appears in the second author's Ph.D. dissertation written at Indiana University under the supervision of John B. Conway. After completion of Olin's thesis the authors jointly studied antisymmetry for subalgebras of $L^{\infty}(\mu)$. The results of this work led to the remaining theorems in this paper. 
THEOREM 3. If $P^{\infty}(\mu)$ is an antisymmetric algebra then there is a simply connected region $G$ such that $\mu$ is supported by $G^{-}$and there is a natural injection of $H^{\infty}(G)$ into $L^{\infty}(\mu)$ whose image is $P^{\infty}(\mu)$.

Here $H^{\infty}(G)$ denotes the bounded analytic functions on $G$ and the above identification is denoted by $P^{\infty}(\mu)=H^{\infty}(G, \mu)$. If Theorems 2 and 3 are combined then a refinement of Sarason's Theorem 2 in [2] is obtained. Similar results can be obtained for $R^{\infty}(K, \mu)$, the weak star closure of $R(K)$ in $L^{\infty}(\mu)$, for certain compact subsets that carry $\mu$.

For any operator $T$, Red $T$ denotes the lattice of reducing subspaces of $T$.

THEOREM 4. There are spaces $\left\{K_{n}: n \geqslant 0\right\}$ in $\operatorname{Red} N$ and $\left\{H_{n}: n \geqslant 0\right\}$ in Red $S$ such that:

(a) $\stackrel{H}{\prime}_{n} \subseteq K_{n}$ and $N$ leaves $H_{n}$ invariant;

(b) $K=K_{0} \oplus K_{1} \oplus \cdots$ and $H=H_{0} \oplus H_{1} \oplus \cdots$;

(c) $K_{0}=H_{0}$ and $N \mid H_{0}$ is a reductive normal operator;

(d) $\mathrm{A}\left(S \mid \mathrm{H}_{n}\right)$ is antisymmetric for $n \geqslant 1$;

(e) $N \mid K_{n}$ is the minimal normal extension of $S \mid H_{n}$ for $n \geqslant 1$;

(f) $\mathrm{A}(S)=\mathrm{A}\left(S \mid H_{0}\right) \oplus \mathrm{A}\left(S \mid H_{1}\right) \oplus \cdots$ and $\mathrm{A}(N)=\mathrm{A}\left(N \mid K_{0}\right) \oplus \mathrm{A}\left(N \mid K_{1}\right)$ $\oplus \cdots \cdot$

The effect of Theorem 4 is to reduce the study of subnormal operators to the case where $A(S)$ is antisymmetric.

THEOREM 5. If $P^{\infty}(\mu)$ is antisymmetric and $f \in P^{\infty}(\mu)$ is not constant then $f(N)$ is the minimal normal extension of $f(S)$.

If the hypothesis of antisymmetry in the preceding theorem is deleted then Theorems 4 and 5 can be combined to determine the minimal normal extension of $f(S)$ for any $f$ in $P^{\infty}(\mu)$.

Theorem 6. If $P^{\infty}(\mu)$ is antisymmetric, $P^{\infty}(\mu)=H^{\infty}(G, \mu)$, and $f \in P^{\infty}(\mu)$ then

$$
\sigma(f(S))=[f(\sigma(S) \cap G)]^{-} \cup \sigma(f(N))
$$

An application of the above results and the techniques used to prove them gives the following.

THEOREM 7. If $A$ is any nonreductive normal operator on $K$ then there is a nonreducing invariant subspace $H$ for $A$ such that $A$ is the minimal normal extension of $A \mid \mathrm{H}$ and $A \mid \mathrm{H}$ has a nontrivial invariant subspace.

It is also possible to define a functional calculus for $S$ using functions in $R^{\infty}(\sigma(S), \mu)$. With certain restrictions on $S$ the methods used to obtain the preceding theorems yield analogous results. Work is in progress to remove these restrictions. 
The details of this work as well as further refinements, technical information, and applications will appear elsewhere.

\section{REFERENCES}

1. J. Bram, Subnormal operators, Duke Math. J. 22 (1955), 75-94. MR 16, 835.

2. D. Sarason, Weak-star density of polynomiais, J. Reine Angew. Math. 252 (1972), 1-15. MR $45 \# 4156$.

DEPARTMENT OF MATHEMATICS, INDIANA UNIVERSITY, BLOOMINGTON, INDIANA 47401

DEPARTMENT OF MATHEMATICS, VIRGINIA POLYTECHNICAL INSTITUTE, BLACKSBURG, VIRGINIA 24060 